\title{
MULTIDIMENSIONAL SIGNAL RECONSTRUCTION FROM MULTICHANNEL ACQUISITION
}

\author{
Ka L. Law, Robert M. Fossum \\ Department of Mathematics \\ University of Illinois at Urbana-Champaign \\ Urbana IL 61801
}

\author{
$\operatorname{Minh} N$. Do \\ Department of Electrical \\ and Computer Engineering \\ University of Illinois at Urbana-Champaign \\ Urbana IL 61801
}

\begin{abstract}
We provide an analysis of the algorithms necessary for the optimal use of multidimensional signal reconstruction from multichannel acquisition. Firstly, we provide computable conditions to test the matrix invertibility and propose algorithms to find a particular inverse. Secondly, we determine the existence of perfect reconstruction systems for given FIR analysis filters with some sampling matrices and some FIR synthesis polyphase matrices. Then, we present the development of an efficient algorithm designed to find a sampling matrix with maximum sampling rate and FIR synthesis polyphase matrix for given FIR analysis filters so that the system provides a perfect reconstruction. Once a particular synthesis matrix is found, we can characterize all synthesis matrices and find an optimal one according to a design criterion.
\end{abstract}

Index Terms - Inverse Matrix Problem, Hermite Normal Form, Smith Normal Form,Perfect Reconstruction, Multichannel convolution.

\section{INTRODUCTION}

In recent decades, multirate systems have become important applications in several engineering areas. The ideas of multirate systems have been extended from one dimensional systems to multidimensional systems [1]. The most popular multidimensional multirate system is $M$-dimensional $N$-channel perfect reconstruction (PR) finite impulse response (FIR) uniform filter bank (FB) system [2, 3].

In the traditional setting, the analysis filters and the sampling matrix are given in the uniform filter bank system. The goal is to find synthesis filters such that the system remains a perfect reconstruction for all input signals. In this paper, we relax the restriction that only the analysis filters are given. The new goal is to find a suitable sampling matrix and synthesis polyphase matrix which satisfy a perfect reconstruction condition. Suppose that we have an $N$-channel convolution system in $M$ dimensions. Instead of taking all the data and applying multichannel deconvolution, we can first reduce the collected data set by a sampling matrix $\boldsymbol{D}$ and still perfectly reconstruct the signal with a PR synthesis polyphase matrix. Of course, we want the sampling factor to be as large as possible because it would give us a minimum collected data set. To address this situation, we want to answer the following questions:

Problem 1 Given analysis filters, can we have a PR system with some sampling matrix $\boldsymbol{D}$ and FIR synthesis polyphase matrix?

Problem 2 If so, can we find a sampling matrix $\boldsymbol{D}$ having a maximal sampling factor and a particular FIR synthesis polyphase matrix? Can we have an algorithm to do so?

These questions can be approached differently in one dimensional and multidimensional cases. If they are one dimensional filter banks, then the answers to the problems are trivial. Since $\boldsymbol{D}$ is $1 \times 1$ matrix, we just need to do a linear search on sampling factors. If they are multidimensional systems, however, then there are an infinite many matrices with a given sampling factor. We will address these two problems after we state Hermite normal form and Smith normal form.

Once we find a maximal sampling matrix and a particular synthesis mattrix, we provide an algorithm to obtain an optimal FIR synthesis polyphase matrix according to a design criterion.

\section{PROBLEM FORMULATION}

Let $M, N$ and $\boldsymbol{D}$ be the dimension of signals, the number of channels and an $M \times M$ sampling matrix with integer entries respectively. Let $P$ be the sampling factor at each channel, $P:=|\operatorname{det} \boldsymbol{D}|$. Let $\mathcal{N}(\boldsymbol{D})$ be the quotient group of $\boldsymbol{D} \mathbb{Z}^{M}$ in $\mathbb{Z}^{M}$. Without loss of generality, we make use of Vaidyanathan's definition [1] and denote $\left[\boldsymbol{l}_{j}\right]$ to be $\boldsymbol{l}_{j}+$ $\boldsymbol{D} \mathbb{Z}^{M} \in \mathcal{N}(\boldsymbol{D})$ where $\boldsymbol{l}_{j} \in\{\boldsymbol{D} \boldsymbol{t} \mid \boldsymbol{t} \in[0,1)\}$. By polyphase decomposition (PD), the analysis and synthesis parts can be represented by an $N \times P$ matrix $\boldsymbol{H}(\boldsymbol{z})$ and $P \times N$ matrix $\boldsymbol{G}(\boldsymbol{z})$ shown in Fig. 1(b) and Fig. 1(c), respectively, where 


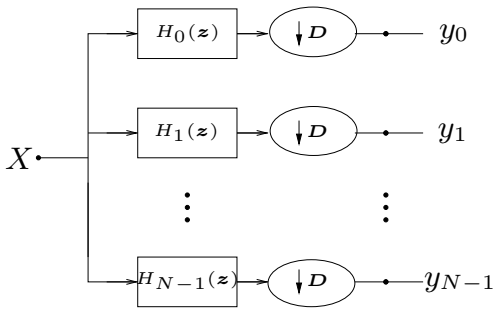

(a)

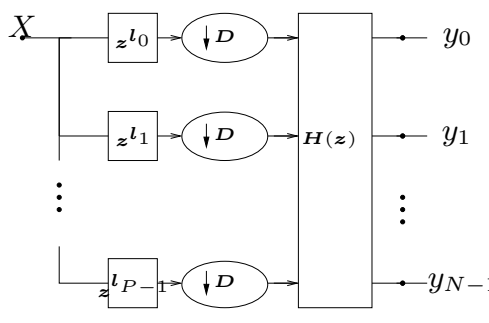

(b)

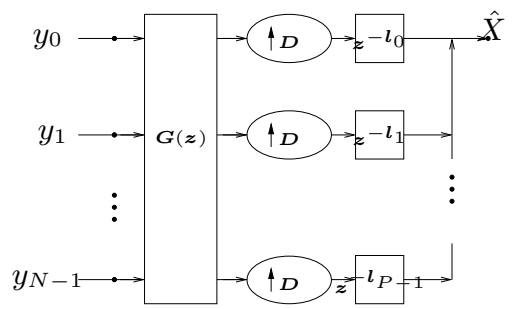

(c)

Fig. 1. (a) Analysis Part: Multichannel convolution followed by downsampling $D$. (b) Polyphase representation of the analysis part. (c) Synthesis polypahse reconstrcution.

every element is a Laurent polynomial in $z$. The PR condition $\hat{X}(\boldsymbol{z})=X(\boldsymbol{z})$ is equivalent to $\boldsymbol{G}(\boldsymbol{z}) \boldsymbol{H}(\boldsymbol{z})=\boldsymbol{I}_{P}$.

The data acquisition can be modeled shown in Fig. 1(a). We consider $H_{i}(\boldsymbol{z})$ fixed (i.e. point spread function of sampling devices), but the sampling matrix $\boldsymbol{D}$ can be changed. We provide an efficient algorithm to find $\boldsymbol{D}$ having maximal sampling rate and FIR synthesis polyphase matrix $\boldsymbol{G}(\boldsymbol{z})$ such that PR condition hold. This is a generalization of multichannel deconvolution problem. (i.e. $\boldsymbol{D}=I$ )

\section{INVERSE MATRIX PROBLEM}

We can generalize Proposition 2 from [4], which considers the case $P=1$, so that we can determine whether an $N \times P$ polynomial matrix is invertible or not. For the proof of the following two propositions, please refer to our preprint [3].

Proposition 1 Suppose $\boldsymbol{H}(\boldsymbol{z})$ is an $N \times P$ polynomial matrix. Let $S=\left\langle\boldsymbol{h}_{1}(\boldsymbol{z}), \ldots ., \boldsymbol{h}_{N}(\boldsymbol{z})\right\rangle$ be the $\mathbb{C}[\boldsymbol{z}]$-submodule of $\mathbb{C}[\boldsymbol{z}]^{P}$ generated by the rows $\boldsymbol{h}_{i}(\boldsymbol{z})$ of $\boldsymbol{H}(\boldsymbol{z})$. Then $\boldsymbol{H}(\boldsymbol{z})$ is invertible if and only if the reduced Gröbner basis of $S$ is $\left\{\boldsymbol{e}_{i}\right\}_{i=1, \ldots, P}$ where $\boldsymbol{e}_{i}$ is the $i$-th row of the $P \times P$ identity matrix.

Proposition 2 Suppose $\boldsymbol{H}(\boldsymbol{z})$ is an $N \times P$ Laurent polynomial matrix. Consider the $(N+P) \times P$ matrix $\boldsymbol{H}^{\prime}(\boldsymbol{z}, w)=$ $\left(\begin{array}{c}\boldsymbol{z}^{\boldsymbol{m}} \boldsymbol{H}(\boldsymbol{z}) \\ \left(1-z_{1} z_{2} \ldots z_{M} w\right) \boldsymbol{I}_{P}\end{array}\right)$ where $\boldsymbol{m} \in \mathbb{N}^{M}$ is such that $\boldsymbol{z}^{\boldsymbol{m}} \boldsymbol{H}(\boldsymbol{z})$ is a polynomial matrix, $w$ is a new variable, and $I_{P}$ is a $P \times P$ identity matrix. Then $\boldsymbol{H}(\boldsymbol{z})$ is Laurent polynomial left invertible if and only if $\boldsymbol{H}^{\prime}(\boldsymbol{z}, w)$ is a polynomial left invertible.

From Proposition 1 and 2, we introduce two new algorithms to generate an inverse matrix by using Gröbner bases if the given matrix is invertible.

Algorithm 1 (Particular Polynomial Inverse) The computational algorithm for a polynomial left inverse matrix. Input: $N \times P$ polynomial $M$-variate matrix $\boldsymbol{H}(\boldsymbol{z})$. Output: $P \times N$ polynomial matrix $\boldsymbol{G}(\boldsymbol{z})$, if it exists.
1. Compute the reduced Gröbner basis of $\left\{\boldsymbol{h}_{1}(\boldsymbol{z}), \ldots, \boldsymbol{h}_{N}(\boldsymbol{z})\right\}$ where $\boldsymbol{h}_{i}(\boldsymbol{z})$ is a row of $\boldsymbol{H}(\boldsymbol{z})$ and the associated transformation matrix $\boldsymbol{W}(\boldsymbol{z})$ by using Buchberger's algorithm.

2. If the reduced Gröbner basis is $\left\{\boldsymbol{e}_{i}\right\}_{i=1, . ., P}$, then output $\boldsymbol{W}(\boldsymbol{z})$. Otherwise, there is no solution.

Algorithm 2 (Particular Laurent Polynomial Inverse) The computational algorithm for a Laurent polynomial left inverse matrix.

Input: $N \times P$ Laurent polynomial $M$-variate matrix $\boldsymbol{H}(\boldsymbol{z})$. Output: $P \times N$ Laurent polynomial matrix $\boldsymbol{G}(\boldsymbol{z})$, if it exists. 1. Multiply $\boldsymbol{H}(\boldsymbol{z})$ by a common monomial $\boldsymbol{z}^{\boldsymbol{m}}$ such that $\boldsymbol{H}^{\prime}(\boldsymbol{z}, w)$ is polynomial matrix from Proposition 2.

2. Call Algorithm 1 with input $\boldsymbol{H}^{\prime}(\boldsymbol{z}, w)$.

3. If the output of Algorithm 1 is $\boldsymbol{G}^{\prime}(\boldsymbol{z}, w)$, then output

$$
\boldsymbol{z}^{-\boldsymbol{m}}\left(\boldsymbol{G}_{i j}^{\prime}\left(\boldsymbol{z}, \prod_{k=1}^{M} z_{k}^{-1}\right)\right)_{i=1, \ldots, P ; j=1, \ldots, N} .
$$

Otherwise, there is no solution.

\section{PROPOSED ALGORITHM}

Theorem 1 (Hermite Normal Form) [5] Given an $M \times M$ nonsingular integer-valued matrix $\boldsymbol{D}$, there exists an $M \times M$ unimodular matrix $\boldsymbol{K}$ such that $\boldsymbol{D K}=\boldsymbol{H}$, the Hermite normal form of $\boldsymbol{D}$ whose entries satisfy $h_{i j}=0$ for $j>i$, $h_{i i}>0$ for all $i$ and $0 \leq h_{i j}<h_{i i}$ for $j<i$. Define $C(P)$ to be a set of Hermite normal forms for a given absolute determinant $P$. Then, $\left|C_{1}(P)\right|=1$ and $\left|C_{M}(P)\right|=$ $\sum_{q \mid P} q\left|C_{M-1}(q)\right|$ for $M \geq 2$ where $\left|C_{M}(P)\right|$ is defined to be the size of $C_{M}(P)$.

When $M=2$, then we have $\left|C_{2}(P)\right|=\sum_{q \mid P} q$. Robin [6] proves that $\left|C_{2}(P)\right|=\mathrm{O}(P \log \log P)$.

For the following algorithm, we will employ the Smith normal form. For a detail discussion, please refer to [5]. There are many algorithms for efficiently computing the Smith 
normal form. Among all algorithms, The Storijohann's algorithm using modular techniques to compute Smith normal form and transformation matrices gives the best known complexity analysis for the integer matrices [7].

Corollary 1 [8] Let $\boldsymbol{D}=\boldsymbol{U} \boldsymbol{\Lambda} \boldsymbol{V}$ be a Smith normal form. Define $\Phi(\boldsymbol{l})=\boldsymbol{U} \boldsymbol{l}$. Then $\mathcal{N}(\boldsymbol{D})=\Phi(\mathcal{N}(\boldsymbol{\Lambda}))$.

Algorithm 3 The algorithm of finding a sampling matrix and synthesis filters satisfying the PR condition for the input sampling rate.

Input: FIR analysis filters $H_{i}(\boldsymbol{z})$ and a sampling rate $P$.

Output: sampling matrix $D$ and FIR synthesis polyphase matrix together with the set $\mathcal{N}(D)$, if it exists.

1. If $C(P)$ is empty, then there is no solution.

2. Take $\boldsymbol{D}$ from $C(P)$ and set $C(P):=C(P)-\{\boldsymbol{D}\}$.

3. Let $\boldsymbol{D}=\boldsymbol{U} \boldsymbol{\Lambda} \boldsymbol{V}$ be a Smith normal form. Find $\mathcal{N}(\boldsymbol{D})$ by Corollary 1.

4. By the $P B, \boldsymbol{H}(\boldsymbol{z})$ is an analysis polyphase matrix corresponding to $H_{i}(\boldsymbol{z})$ and $\boldsymbol{D}$.

5. Find a left inverse $\boldsymbol{G}(\boldsymbol{z})$ of $\boldsymbol{H}(\boldsymbol{z})$ by Algorithm 2. Otherwise goto 1 .

6. Output the FIR synthesis polyphase matrix $G(z)$ together with the set $\mathcal{N}(\boldsymbol{D})$ and the sampling matrix $\boldsymbol{D}$.

For the proof of the following Theorems and Lemma, please refer to our preprint [8].

Theorem 2 The Polynomial analysis filters $H_{i}(\boldsymbol{z})$ have no common solution if and only if the system provide a PR for some sampling matrix and some polynomial synthesis polyphase matrix.

Theorem 3 Every common solution of the FIR analysis filters $H_{i}(\boldsymbol{z})$ has at least one zero component if and only if the system is provide a PR for some sampling matrix and some FIR synthesis polyphase matrix.

This theorem addresses the first problem in the beginning of this paper. To determine whether the system is perfectly reconstructable for given a set of analysis filters, we only need to examine the solution set of the analysis filters. Now we provide an upper bound of the search method.

Lemma 1 Let $H_{0}(\boldsymbol{z}), H_{1}(\boldsymbol{z}), \ldots, H_{N-1}(\boldsymbol{z})$ be analysis filters. If $P>N$, then there not exists any sampling matrix $D$ and any synthesis polyphase matrix such that the system provides a PR.

According to above Lemma and Hermite normal form, the search space for sampling matrices to determine the perfect reconstructability is a finite process. We propose an algorithm to answer the second problem mentioned in the beginning of the paper.

Algorithm 4 The algorithm of finding a sampling matrix with maximal sampling factor and synthesis filters satisfying the
PR condition.

Input: FIR analysis filters.

Output: sampling matrix $D$ and FIR synthesis polyphase matrix together with the set $\mathcal{N}(D)$, if it exists.

1. Check the solution set of $H_{i}(\boldsymbol{z})$ satisfying the condition of Theorem 3. Otherwise there is no solution.

2. Set $P=N$.

3. Call Algorithm 3 and output FIR synthesis polyphase matrix, the set $\mathcal{N}(\boldsymbol{D})$ and a sampling matrix $\boldsymbol{D}$.

4. Otherwise, set $P=P-1$ and go to 3 .

By Theorem 1, the maximum number of iterations is $\sum_{m=1}^{N}$ $\sum_{q \mid m} q \cdot\left|C_{M-1}(q)\right|$ for $M \geq 2$. When $M=2$, then $\sum_{m=1}^{N}$ $\sum_{q \mid m} q=\frac{\pi^{2}}{12} N^{2}+\mathrm{O}(N \ln N)$ by Handy and Weight [9]. To improve the performance, we can reduce the upper bound $P$ by setting to $N-M$ due to the fact that the possibility of being a PR system is zero when $N-M<P$ [3].

Example 1 Let $H_{0}(\boldsymbol{z})=\left(1+z_{1}\right)\left(1+z_{2}\right), H_{1}(\boldsymbol{z})=(1-$ $\left.z_{1}\right)\left(1-z_{1} z_{2}\right), H_{2}(\boldsymbol{z})=\left(1-z_{1}\right)\left(z_{1}-z_{2}\right), H_{3}(\boldsymbol{z})=(1-$ $\left.z_{2}\right)\left(1-z_{1} z_{2}\right), H_{4}(\boldsymbol{z})=\left(1-z_{2}\right)\left(z_{1}-z_{2}\right), H_{5}(\boldsymbol{z})=(1-$ $\left.z_{1}\right)\left(1-z_{2}\right)$ be the analysis filters. By the Algorithm 4 , we can show that the solution set of analysis filters is an empty set, which implies the system is polynomial perfectly reconstructable. We then obtain a sampling matrix $\boldsymbol{D}=\left(\begin{array}{cc}1 & 0 \\ -2 & 3\end{array}\right)$ with maximum sampling rate and synthesis matrix $\boldsymbol{G}(\boldsymbol{z})=$ $\left.\begin{array}{ccc}-\frac{z_{1}}{8}+\frac{3}{8} & -\frac{z_{1} z_{2}}{24}-\frac{z_{1}}{12}+\frac{z_{2}}{8}+\frac{1}{4} & -\frac{z_{1} z_{2}}{12}-\frac{z_{1}}{24}+\frac{z_{2}}{4}+\frac{1}{8} \\ \frac{1}{4} & \frac{z_{2}}{12}+\frac{1}{2} & \frac{z_{2}}{6}+\frac{3}{4} \\ 0 & \frac{z_{2}}{3} & \frac{2 z_{2}}{3} \\ \frac{1}{4} & \frac{z_{1}}{3}+\frac{z_{2}}{12}-\frac{1}{2} & \frac{2 z_{1}}{3}+\frac{z_{2}}{6}-\frac{5}{4} \\ 0 & -\frac{2 z_{1}}{3} & -\frac{4 z_{1}}{3}+1 \\ \frac{z_{1}}{8}+\frac{1}{8} & \frac{z_{1} z_{2}}{24}-\frac{z_{1}}{4}-\frac{7 z_{2}}{24}-\frac{1}{4} & \frac{z_{1} z_{2}}{12}-\frac{5 z_{1}}{8}-\frac{7 z_{2}}{12}+\frac{3}{8}\end{array}\right)$

\section{OPTIMIZATION OF SYNTHESIS FIR POLYPHASE MATRIX}

By Zhou and Do [10], we can characterize the set of all FIR synthesis matrices $\{\boldsymbol{G}(\boldsymbol{z}) \mid \boldsymbol{G}(\boldsymbol{z})=\tilde{\boldsymbol{G}}(\boldsymbol{z})+\boldsymbol{A}(\boldsymbol{z})(\boldsymbol{I}-\boldsymbol{H}(\boldsymbol{z})$ $\tilde{\boldsymbol{G}}(\boldsymbol{z})), \boldsymbol{A}(\boldsymbol{z})$ is an arbitrary $P \times N$ Laurent polynomial matrix $\}$ from a particular synthesis matrix $\tilde{\boldsymbol{G}}(\boldsymbol{z})$. By using the characterization and extending Agorithm 2 by Zhou and Do [4], we have the following algorithm: (Please refer to our preprint [3] for the detail discussion)

Algorithm 5 (Optimal Inverse) The computational algorithm for an optimal left inverse matrix by minimizing the mean square error for a given support $Q$ of $\boldsymbol{A}(\boldsymbol{z})$ where $A_{i j}(\boldsymbol{z})=$ $\sum_{\boldsymbol{k} \in Q} a_{i j, \boldsymbol{k}} z^{\boldsymbol{k}}$.

Input: $N \times P$ matrix $\boldsymbol{H}(\boldsymbol{z})$ with $M$ variables.

Output: an optimal inverse $P \times N$ matrix $\boldsymbol{G}(\boldsymbol{z})$ with respect to the given support.

1. Use Algorithm 2, to compute a particular inverse $\tilde{\boldsymbol{G}}(\boldsymbol{z})$.

2. Compute $\boldsymbol{G}_{i}(\boldsymbol{z})$ the ith rows of $\boldsymbol{G}(\boldsymbol{z})$ with respect to $a_{i j, \boldsymbol{k}}$. 


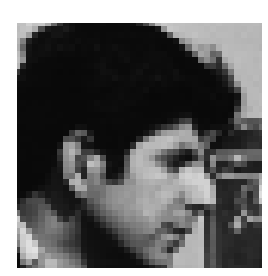

(a)

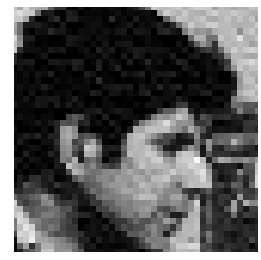

(b)

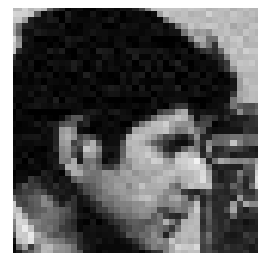

(c)

Fig. 2. The origin image and the reconstruction outputs are imposed by additive white noise with $\sigma=4$. (a) The original image. (b) Algorithm 1, MSE=127.42. (c) Algorithm 5, MSE=65.07.

3. For each $i$, compute $\operatorname{MSE}\left(a_{i j, \boldsymbol{k}}\right)=\sigma^{2} \sum_{j=1}^{K} \sum_{\boldsymbol{k} \in Q}\left|g_{i j, \boldsymbol{k}}\right|^{2}$. 4. Set all partial derivatives of $\operatorname{MSE}\left(a_{i j, \boldsymbol{k}}\right)$ with respect to $a_{i j, k}$ to zero and solve the linear equations.

5. Back substitute $\left\{a_{i j, \boldsymbol{k}}\right\}$ in $\boldsymbol{G}(\boldsymbol{z})$ to obtain an optimal solution and output $\boldsymbol{G}(\boldsymbol{z})$.

\subsection{Simulation}

We demonstrate a simulation of 4-channel acquisition with the subband signals containing different levels of white Gaussian noise. In each subband signal, the white Gaussian noise is channel independently with zero mean and power density $\sigma^{2}$. In the simulation, we assume the input signal is a 2-dimensional image shown in Fig. 2(a). Given analysis filters $H_{1}(\boldsymbol{z})=$ $1 / 3 z_{1} z_{2}+z_{2}+z_{1}^{-1}, H_{2}(\boldsymbol{z})=1 / 3 z_{1} z_{2}+1 / 3 z_{1} z_{2}^{-1}+z_{2}$, $H_{3}(\boldsymbol{z})=1+z_{2}+z_{1}^{-1}, H_{4}(\boldsymbol{z})=1 / 3 z_{1} z_{2}$, and a quincunx sampling matrix $\boldsymbol{D}=\left(\begin{array}{cc}1 & 1 \\ 1 & -1\end{array}\right)$. In the experiment, we want to compare with reconstruction performance in MSE between Algorithm 1 and Algorithm 5. The reconstruction images are shown in Fig. 2(b) - 2(c) with $\sigma=4$. We find out that for different levels of white noise, Algorithm 5 has a better performance in MSE than Algorithm 1. Another observation is that when $\sigma$ is below 4 , the reconstruction has relatively good quality observed by eyes.

\section{CONCLUSION}

In this paper, first we handle the inverse matrix problem. Then we address the necessary and sufficient condition on the analysis filters to have a PR system with some sampling matrix and some synthesis matrix. Using Hermite and Smith normal form, we propose an efficient algorithm to find a FIR PR synthesis polyphase matrix and a sampling matrix such that collected data is the minimum among all sampling matrices up to equivalence. Once having a particular synthesis matrix, we can parametrize the set of all synthesis matrices where an optimal solution can be found for a given design criterion.

\section{REFERENCES}

[1] P. Vaidyanathan, "New results in multidimensional multirate systems," in IEEE International Sympoisum on Circuits and Systems, Jun 1991, pp. 468-471.

[2] H. Park, "Complete parametrization of synthesis in multidimensional perfect reconstruction FIR systems," IEEE International Symposium on Circuits and Systems, vol. 5, pp. 41-44, 1999.

[3] K. L. Law, R. M. Fossum, and M. N. Do, "Invertibility of multidimensional FIR filter banks and mimo systems," IEEE Trans. Signal Proc., 2008. submitted; available at https://netfiles.uiuc.edu/kalaw/homepage/final.pdf.

[4] J. Zhou and M. N. Do, "Multidimensional multichannel FIR deconvolution using Gröbner bases," IEEE Trans. Image Proc., vol. 15, no. 10, pp. 2998-3007, Oct. 2006.

[5] M. Newman, Integral Matrices. Academic Press, INC., 1972.

[6] G. Robin, "Grandes valeurs de la fonction somme des diviseurs et hypothse de riemann," Journal de Mathmatiques Pures et Appliques, vol. 63, pp. 187-213, 1984.

[7] A. Storjohann, "Nearly optimal algorithms for computing smith normal forms of integer matrices," in Proceedings of ISSAC. ACM Press, 1996, pp. 267-274.

[8] K. L. Law, R. M. Fossum, and M. N. Do, "Multidimensional fir filter banks and sampling matrices," IEEE Trans. Signal Proc., 2008, to be submitted; available at https://netfiles.uiuc.edu/kalaw/homepage/sampling

[9] G. H. Hardy and E. M. Weight, An Introduction to the Theory of Numbers. Oxford University Press, 1979.

[10] J. Zhou and M. N. Do, "Multidimensional oversampled filter banks," in Proc. of SPIE Conference on Wavelet Applications in Signal and Image Processing XI, San Diego, USA, Jul. 2005. 